\title{
Imaging spectrum of acquired uterine vascular abnormalities with angiographic correlates, a pictorial review
}

\author{
Laiba Masood ${ }^{1,2^{*}}$, Atif I. Rana ${ }^{1,2}$, Zahid A. Khan ${ }^{1,2}$, Saman Nosheen ${ }^{1,2}$, Haider Ali $^{1,2}$ and Jamshed Anwar ${ }^{1,2}$
}

\begin{abstract}
Background: Acquired uterine arterial anomalies, including uterine artery pseudoaneurysms (UAP), arteriovenous malformations (AVMs) and arteriovenous fistulae (AFVs), are rare presenting causes of abnormal uterine bleeding. Timely diagnosis is essential for safe and effective treatment, avoiding life-threatening haemorrhage resulting from erroneous uterine curettage due to misdiagnosing these as other more common differentials.

Main text: This pictorial review discusses the ultrasound (USG), CT and MRI features of various acquired uterine vascular abnormalities with angiographic correlates.

Conclusion: Acquired uterine arteriovenous injuries are a fundamental cause of dysfunctional intractable bleeding recalcitrant to traditional conservative management. Endovascular transcatheter uterine artery embolisation is an increasingly popular and safe mode of treatment, especially in young patients desiring to have the option of future pregnancies, with lesser morbidity and in-hospital stay duration.
\end{abstract}

Keywords: Arteriovenous malformation, Ultrasound, Magnetic resonance imaging, Computed tomography angiography, Uterine artery embolisation, Uterus

\section{Background}

The uterus is a richly supplied vascular organ having the uterine artery as its main arterial supply. The ascending branch of uterine artery further gives off arcuate branches, which surround and circumferentially supply the outer and central segments of the myometrium. Radial branches then take off to course through the remaining myometrium inwards to form spiral and basal arteries which supply the endometrium. In its superior course, the ascending branch anastomoses with the branches of ovarian artery. The venous supply to the uterus follows analogously [1].

It is not uncommon to see pelvic vascular lesions in females of reproductive age. Repetitive iatrogenic stresses

\footnotetext{
*Correspondence: laibamasood66@gmail.com

${ }^{1}$ Department of Diagnostic and Interventional Radiology, Shifa

International Hospital, Islamabad, Pakistan

Full list of author information is available at the end of the article
}

like caesarian section and therapeutic dilatation and curettage make this population even more at risk for developing these vascular anomalies. Many women of childbearing age develop post-partum haemorrhage, a known cause of maternal morbidity in developing countries [2,3]. Primarily, the retained products of conception, endometritis or molar pregnancy are the responsible etiologies [2]. Occasionally, uterine vascular abnormalities like AVMs or pseudoaneurysms are also blameworthy for heavy menstrual bleeding $[4,5]$. Although rare, the incidence of uterine vascular lesions like arteriovenous malformations and pseudoaneurysms has been reported quite a few times in the literature, likely due to increased awareness of these pathological injuries and expert diagnostic imaging modalities gynaecologists previously neglected. Attention to the clinical and surgical history with lab profile enables one to narrow down the differential list before instigating appropriate diagnostic imaging and planning a management course. Retained 
products of conception (RPOCs) and gestational trophoblastic disease (GTD) are considerable differentials that may be isolated if there is a history of elevated serum $B$ hCG levels [5, 6]. In that case, acquired uterine vascular abnormalities can be confidently excluded. This pictorial review discusses the ultrasound, CT and MRI features of various acquired uterine vascular abnormalities with angiographic correlations.

\section{Main text \\ Uterine artery pseudoaneurysms}

A defect in the arterial wall due to inadequate sealing during surgery /intervention with resultant bloodfilled sac contained within the adventitia or surrounding perivascular soft tissue is called a pseudoaneurysm. Uterine artery pseudoaneurysm (UAP) is a rare disorder that often occurs after traumatic delivery or traumatic pregnancy termination, including caesarean section or dilatation and curettage [1]. It can also happen after gynaecological interventions, such as myomectomy, hysteroscopy, ovarian puncture or cystectomies [2]. UAPs can present with severe pelvic pain manifesting as one of the causes of post-partum or post-abortion haemorrhage, usually diagnosed after rupture [2,3]. Colour Doppler USG shows the typical Ying yang appearance in a vascular structure with a narrow neck connecting to the adjacent vessel $[3,4]$. Post-contrast CT or MRI in addition to contrast-filled sac may show surrounding pelvic collateralisation [4] (Fig. 1). Urgent treatment is advocated due to the risk of spontaneous rupture or rapid expansion [2].

\section{Uterine artery arteriovenous malformations}

Arteriovenous malformation (AVM) is multiple abnormal communications between the arteries and veins without intervening capillary bed [5]. Uterine AVM is a rare disorder that can be either congenital or acquired (Figs. 2 and 3). Congenital uterine AVMs develop due to defect during embryologic growth with abnormal vascular connections $[5,6]$. The acquired uterine AVM likely occurs due to uterine trauma, such as dilatation and curettage or caesarean delivery. It can also be associated with neoplastic disorders, including gestational trophoblastic disease (GTD) and endometrial adenocarcinoma [7]. Although it is an uncommon entity, it can result in menorrhagia, recurrent abortions and uterine bleeding leading to life-threatening haemorrhaging, which may require blood transfusion $[5,6]$. On grey-scale USG, a bulky uterus may be seen with either a mass or conglomerate of anechoic serpiginous structures, which demonstrate colour filling on Doppler USG, multidirectional high-velocity flow with colour aliasing [8]. CT angiography shows the tangle of vessels with the location, involved vessels and relationship to adjacent structures
[7]. Post-contrast dynamic MRI provides the exact extent of the lesion with endometrial/ myometrial involvement $[7,8]$. Timely diagnosis is vital to prevent complications.

\section{Uterine artery arteriovenous fistulas}

An arteriovenous fistula is a single abnormal direct communication between an artery and a vein [8]. Uterine arteriovenous fistule (AVF) is rare, with fewer cases reported in the literature but a potentially life-threatening condition $[6,8]$. There is no unified consensus regarding the term, and while some describe it separately from AVMs [8], other authors described both as a single entity under the umbrella term AVM or AVM/AVF $[5,9,10]$. These do not have an extrauterine vascular supply or the presence of an intervening capillary network/nidus [8]. Generally, AVFs are post-traumatic/intervention and do not form congenitally [10]. On colour Doppler, AVFs have a high/low resistance arterial waveform and turbulent arterialised venous blood flow with colour aliasing [7]. CT and MRI (Fig. 4) show AVF when early contrast fills the culprit venous vessel on the arterial phase [8].

The combination of any of these vascular abnormalities are even more rare however, may be found coincidingly in the same patient as illustrated (Figs. 5 and 6).

\section{Imaging tools}

Historically, these vascular malformations could only be accurately diagnosed on laparoscopic examination or histopathological analysis of the uterus after hysterectomy [6]. However, now we have access to multiple modalities which can be made use of to locate, determine the size, flow characteristics and relationship to adjacent structures of these vascular abnormalities before making use of a scalpel.

\section{Doppler ultrasound}

Ultrasound is an indispensable and inexpensive imaging aid that is readily available and can accurately diagnose uterine AVMs, AVFs or UAPs [5, 7]. Colour Doppler and spectral analysis provide real-time arterial and venous flow dynamics within these vascular abnormalities and help differentiate between probable differential diagnoses of retained products of conception (RPOCs) in these cases $[5,9,10]$. It also provides rapid assessment, especially by the bedside of patients who may not be haemodynamically stable enough to undergo CT or MRI (Figs. 2 and 4).

\section{CT angiography}

CT angiography with triple phases (arterial, venous and delay) is helpful in the evaluation of these acquired uterine vascular abnormalities and provides adequate 

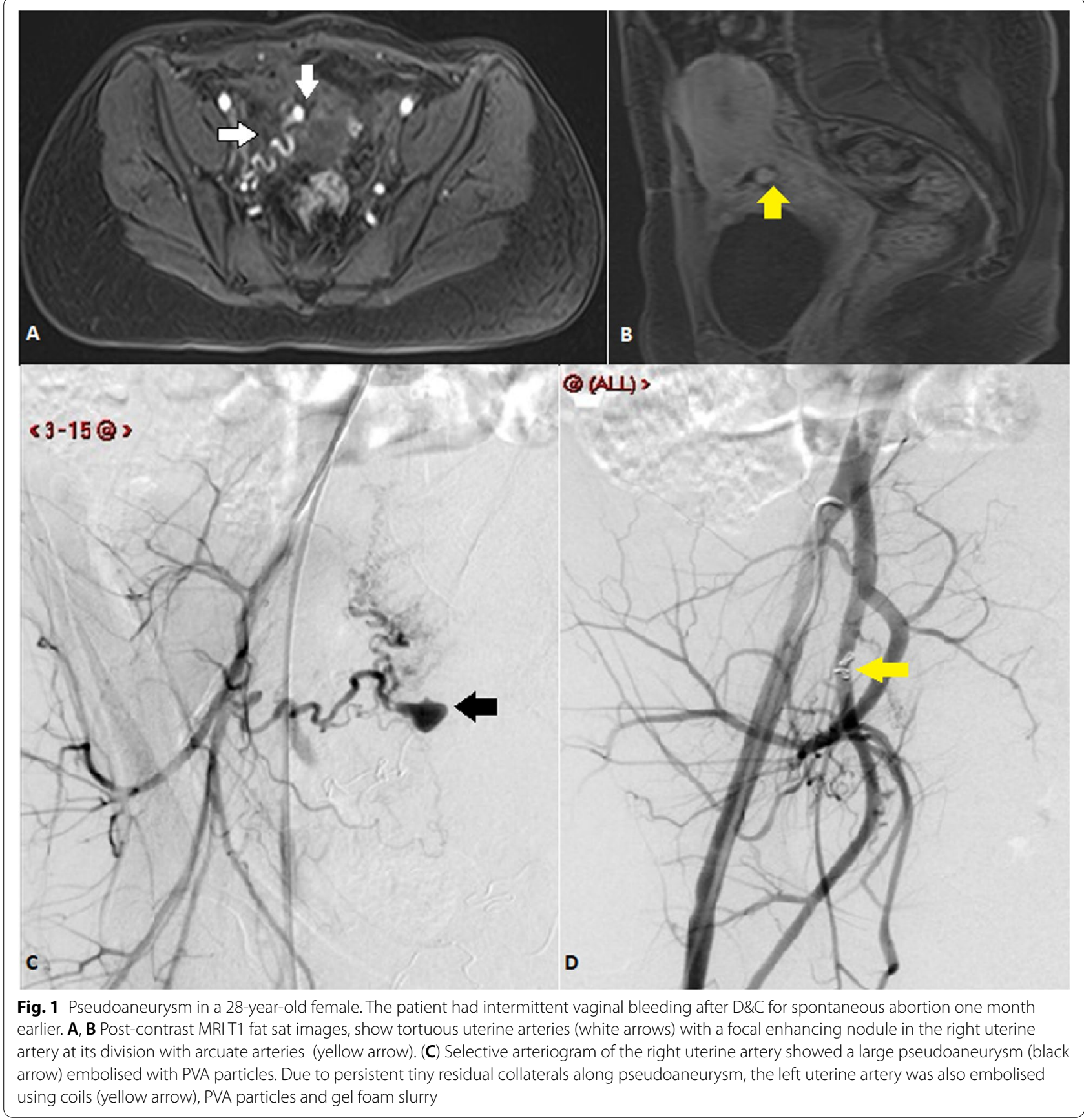

information about the enhancement pattern, locality, possible involved vessels with vascular anatomy [11]. In the case of pseudoaneurysms, it can accurately give the size of the sac and the width of its neck, essential information for the interventional Radiologist for selecting appropriate coils and embolisation agents (Figs. 3 and 5). It is also possible to diagnose active bleeding by contrast extravasation of contrast pooling seen on delayed phase images $[6,12]$. Though ionising radiation slightly reduces its merit, $\mathrm{CT}$ angiography is unquestionably invaluable for emergency diagnosis, rule out an active bleed and decide on the patient's management plan.

\section{MRI with angiography}

Dynamic MRI is a superior imaging modality for the characterisation of vascular abnormalities because of its exceptional contrast resolution, lack of ionising radiation 


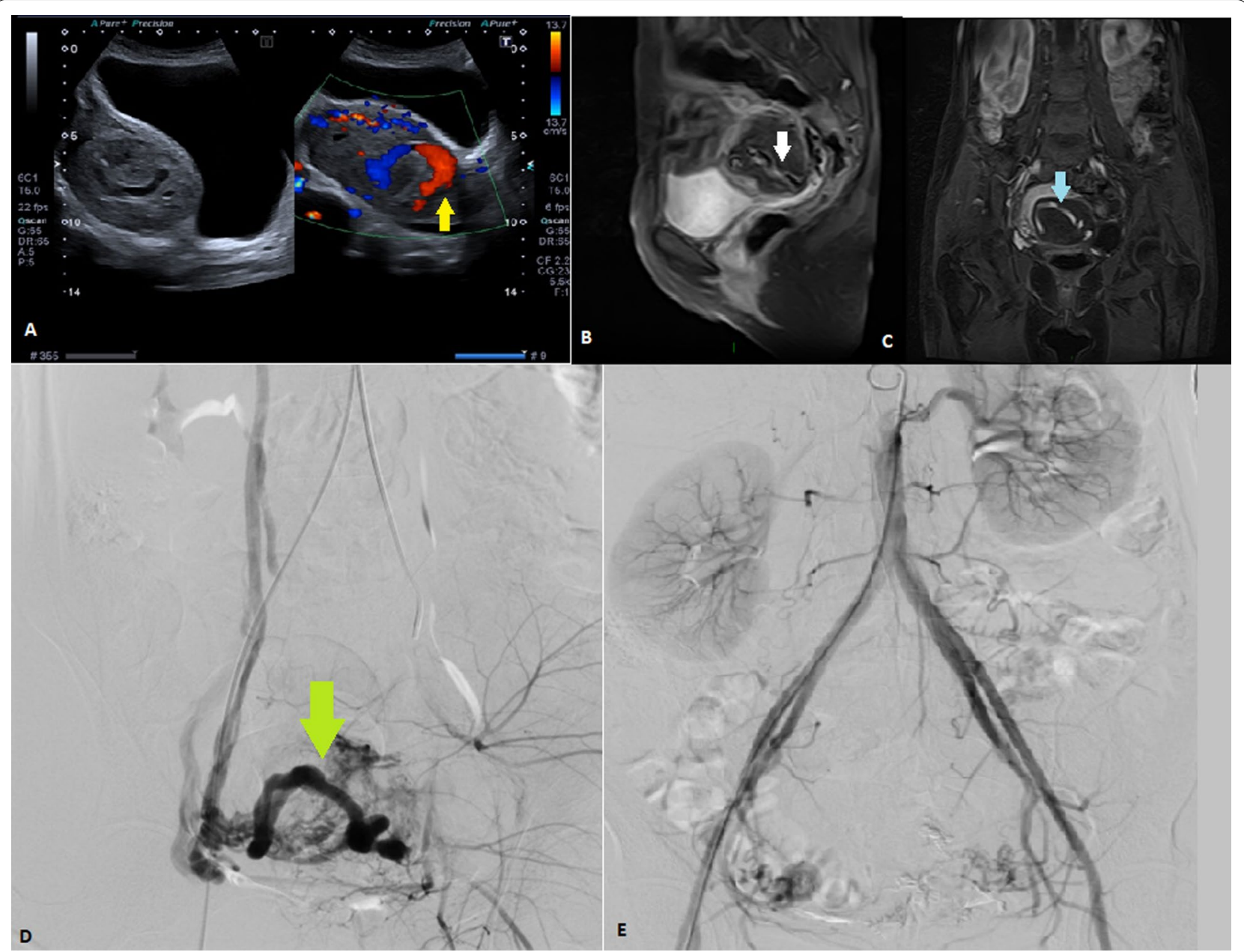

Fig. 2 Acquired uterine AVM in a 31-year-old female. The patient had heaving vaginal bleeding after spontaneous abortion followed by D\&C for two months. A Longitudinal grey-scale US image shows a cystic lesion in the wall of the lower uterus which shows vascularity on Doppler and has varying colours (yellow arrow). Trace fluid is also seen in the cul de sac. B, C Dynamic post-contrast MRI sagittal and coronal sequences show a large tortuous AVM with early draining veins in the lower uterine anterior myometrium (white and blue arrows). D Aortopelvic angiogram shows a large high flow AVM (yellow arrow) in the uterus with early draining veins into the left internal iliac and dilated left ovarian vein into IVC. E Successful left uterine artery histoacryl glue mixed with lipoidol embolisation with satisfactory flow stasis in the AVM

and usefulness in assessing flow dynamics [11]. It also provides a better field of view regarding soft tissue and deep visceral details, which may not be well visualised by $\mathrm{CT}$ or ultrasound due to confounding factors (lack of tissue contrast on CT or bowel gas on ultrasound). MR angiography adds on the vascular anatomical details with an excellent depiction of vascular haemodynamics by clearly delineating arterial inflow from venous drainage and providing information about early venous shunting for appropriate intervention planning $[9,13]$ (Figs. 1 and 6). At the same time, MRI can ensure expert visualisation of local pelvic organs and exclude the presence of other pathologies simultaneously.

\section{Endovascular techniques and embolisation agents}

While digital subtraction angiography remains the gold standard in diagnosing vascular abnormalities, its use is always in conjunction with other diagnostic modalities and is limited to cases requiring definite treatment by endovascular embolisation [13]. It provides excellent details regarding arterial supply to the AVM/AVF, whether unilateral or bilateral, the nidus's size and the draining vein's location $[2,6]$. Early venous shunting into endometrial/myometrial veins can also be expertly identified in cases of AVM/AVF. It also helps locate any collateral vessels, eliminating the risk of procedure's technical and clinical failure. Transcatheter embolisation is an increasingly popular minimally invasive form 


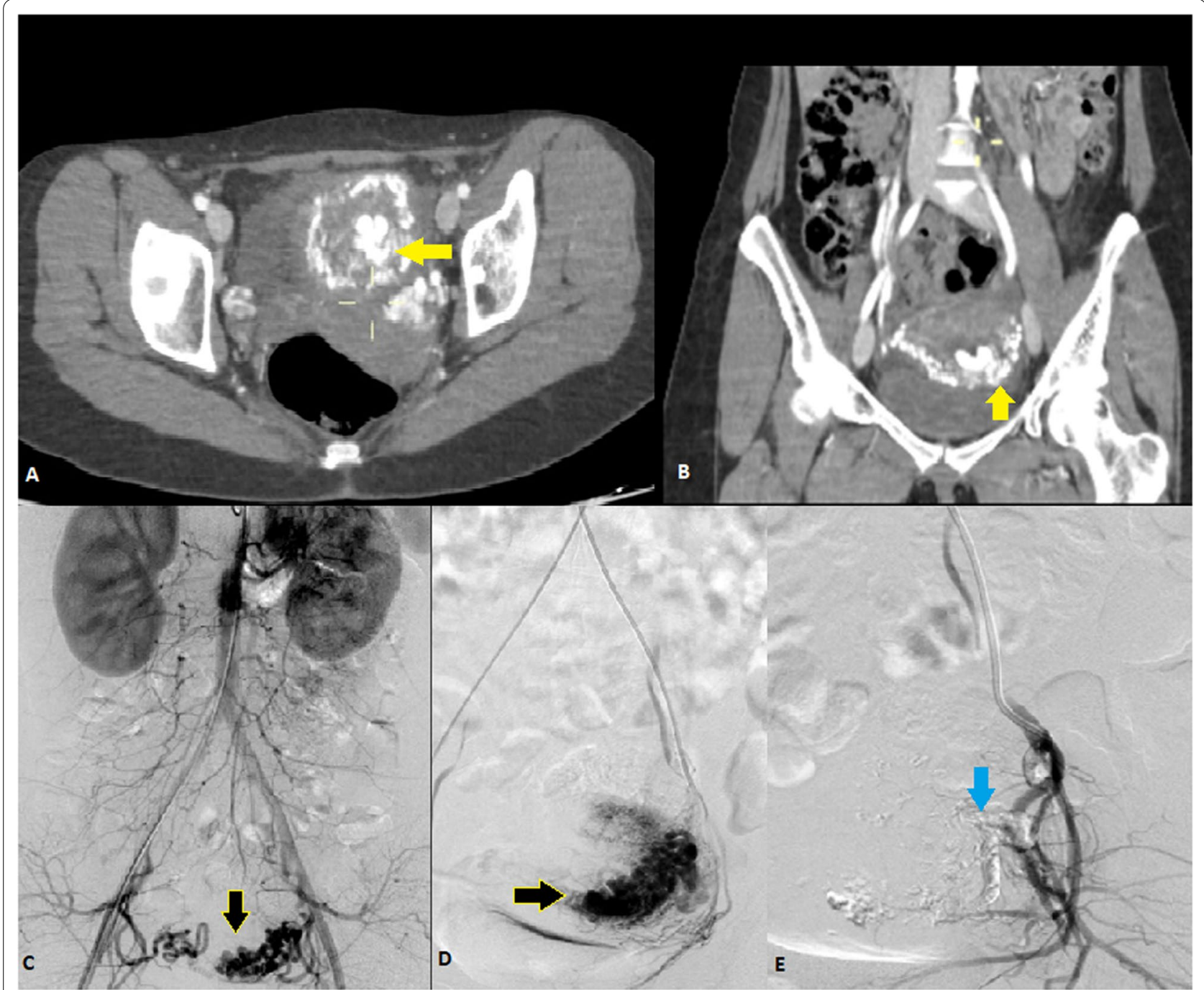

Fig. 3 Uterine AVM in a 28-year-old. The patient had therapeutic D\&C for molar pregnancy, after which she developed invasive GTD followed by methotrexate treatment. She came to us with persistent intermittent vaginal bleeding for one month. The serum B hCG test was negative at the time of presentation. A, B The post-contrast arterial phase image of CT angiogram shows a large dilated tuft of vessels within the posterior aspect of the endometrial canal with extensive myometrial collaterals, suggestive of AVM with nidus within the endometrium (yellow arrow). C, D Angiogram revealed multiple dilated tortuous uterine arterial branches with aneurysmal areas and early filling of veins on both sides (black arrow). E Bilateral uterine artery embolisation was performed with histoacryl glue mixed with lipiodol (blue arrow), followed by satisfactory flow stasis

of treatment for these acquired vascular abnormalities with the advantage of fertility preservation $[14,15]$. It can be super selective, targeting only the arterial feeders involved, i.e. single uterine artery, which successfully eradicates the blood supply to the AVM or UAP, conserving the chance to conceive in the future [14]. Gel foam,
PVA particles, histoacryl glue mixed with lipiodol are the most used agents with coils reserved for the case where there is a risk of shunting embolisation particles into the systemic circulation, or the size of the target vessel is large $[16,17]$.

\section{(See figure on next page.)}

Fig. 4 Uterine AVF in a 28-year-old female. The patient had intermittent vaginal bleeding after D\&C for spontaneous miscarriage three months earlier. A Post-contrast MRI image showed a tortuous enhancing vessel projecting into the myometrium with distended endometrial canal. B Doppler ultrasound images demonstrates aliasing of colour flow in the same area as seen in MRI. C Selective right uterine arteriogram showed large AVF in uterine body supplied by the right uterine artery (yellow arrow). D Embolisation was done with histoacryl glue mixed with lipiodol (black arrow). E Follow-up transvaginal USG examination showed complete resolution of the lesion 

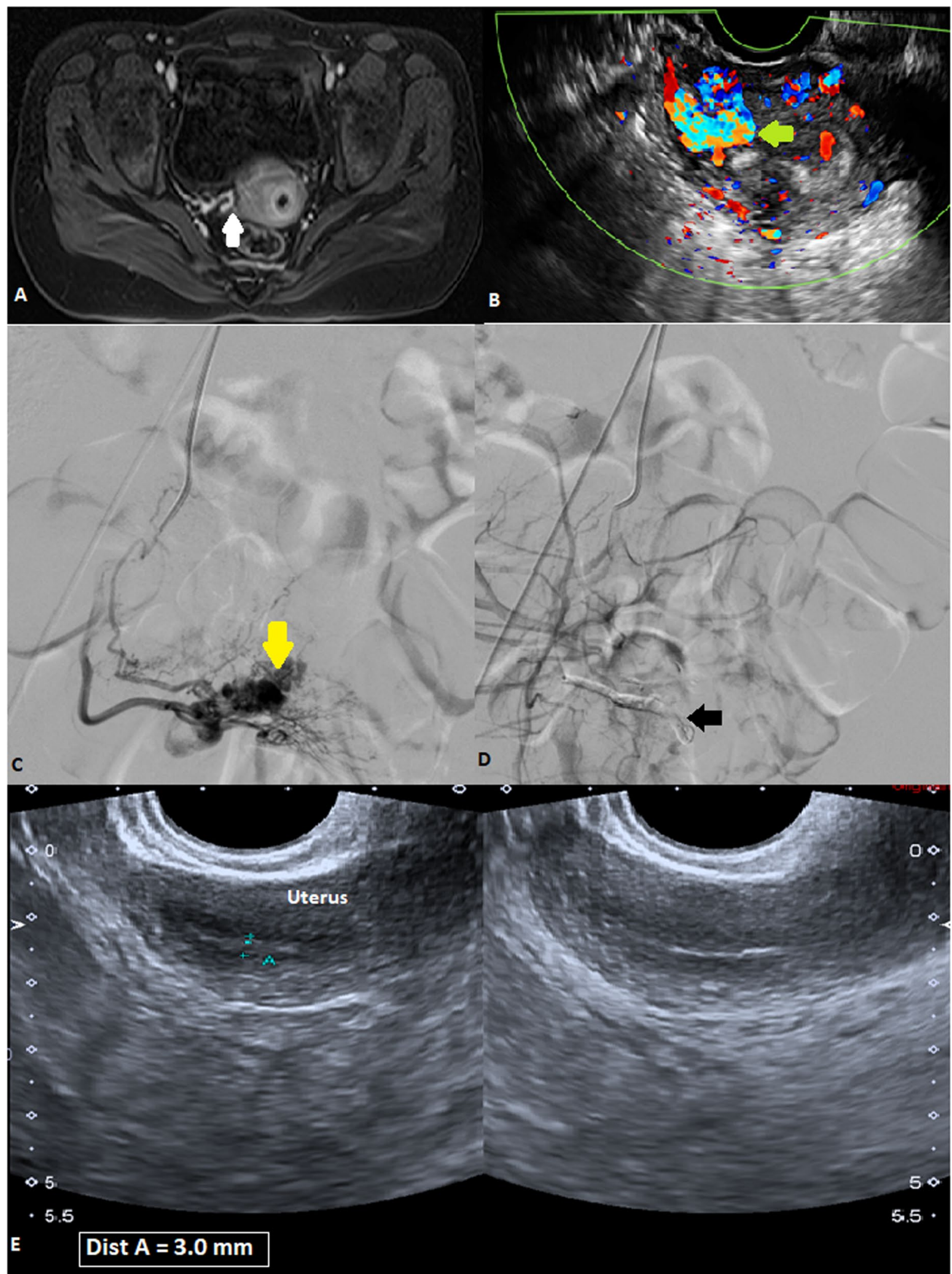

Fig. 4 (See legend on previous page.) 

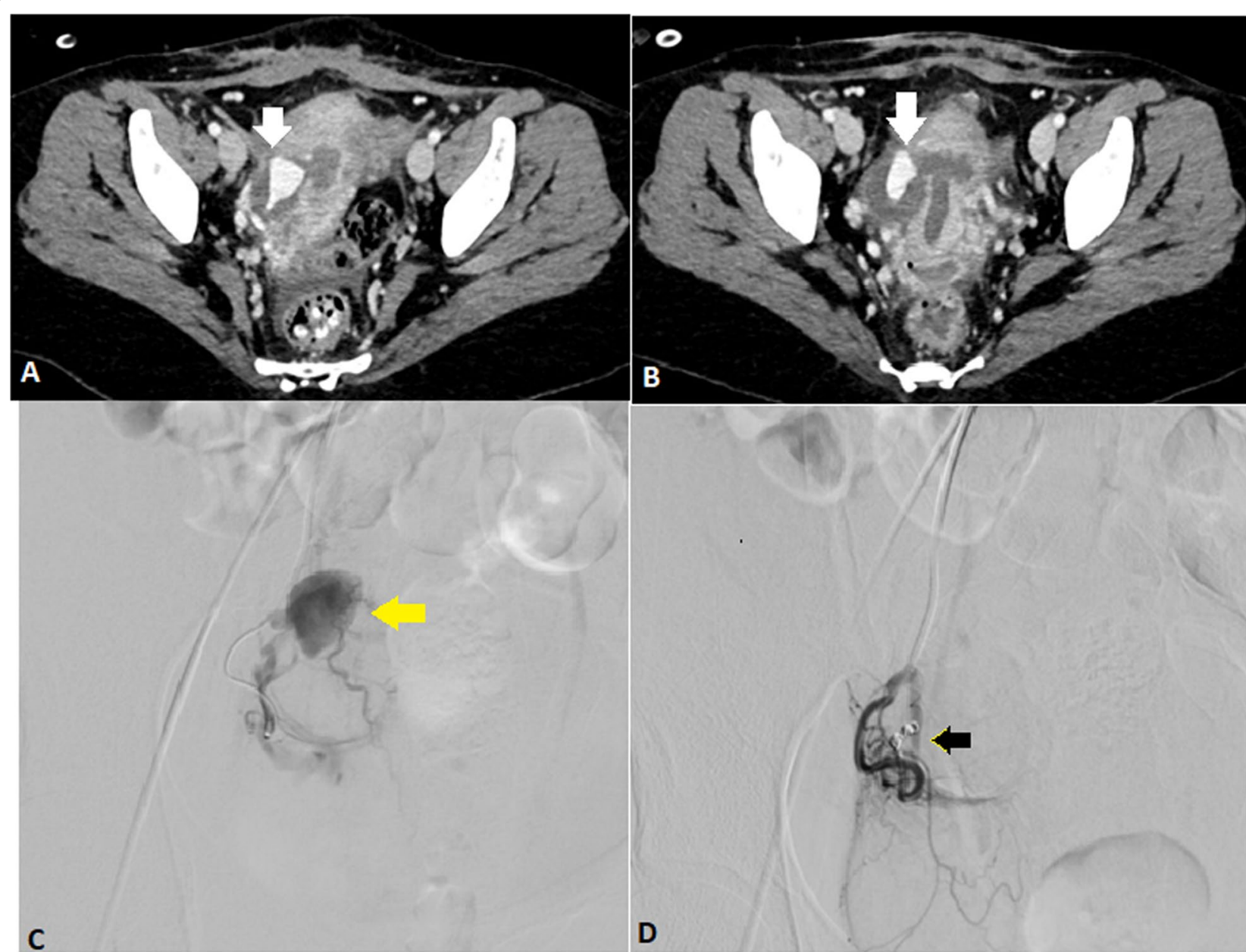

Fig. 5 Acquired pseudoaneurysm and AVM in a 32-year-old. The patient had massive vaginal bleeding after therapeutic D\&C for GTC four weeks earlier. A, B Post-IV contrast CT angiogram image arterial phase showing mildly bulky uterus with partially thrombosed right uterine artery pseudoaneurysm abutting slightly widened scar site in the lower uterine segment (white arrow), with early venous drainage suggesting AVM. C Right internal iliac arteriogram redemonstrated a large narrow neck right uterine artery pseudoaneurysm (yellow arrow) measuring $35 \times 30 \mathrm{~mm}$ with a neck of $2.5 \mathrm{~mm}$. Adjacent arteriovenous shunting with the branches of the right uterine artery suggesting concurrent AVM, was also noted. D Post-embolisation image (performed with the combination of PVA particles and micro-coils (black arrow) since distal/efferent branch could not be selectively cannulated) showed flow stasis without any antegrade or retrograde filling

\section{Follow-up imaging}

Although no specific imaging modality has been endorsed to follow the acquired vascular abnormalities after successful embolisation, it depends entirely on the primary physician. However, Doppler and grey-scale ultrasound seems a sound choice given its role in the assessment of female pelvic organs (Fig. 4) [7, 8]. Similarly, in more complex cases, MRI angiography is superior in depicting the treated vascular abnormality's size, establishing any residual flow to the lesion and possible culprit vessel [11].

\section{Conclusion}

Acquired uterine arteriovenous injuries are a fundamental cause of dysfunctional intractable bleeding recalcitrant to traditional conservative management. In post-partum patients or those who underwent intervention after miscarriage, extreme precaution needs to be taken in diagnosing these vascular abnormalities keeping RPOCs or GTD as differential, which, although more prevalent, will change the entire treatment stance. Endovascular transcatheter uterine artery embolisation is an increasingly popular and safe mode of treatment, especially in young patients desiring to have the option of future pregnancies, with lesser morbidity and in-hospital stay duration. 


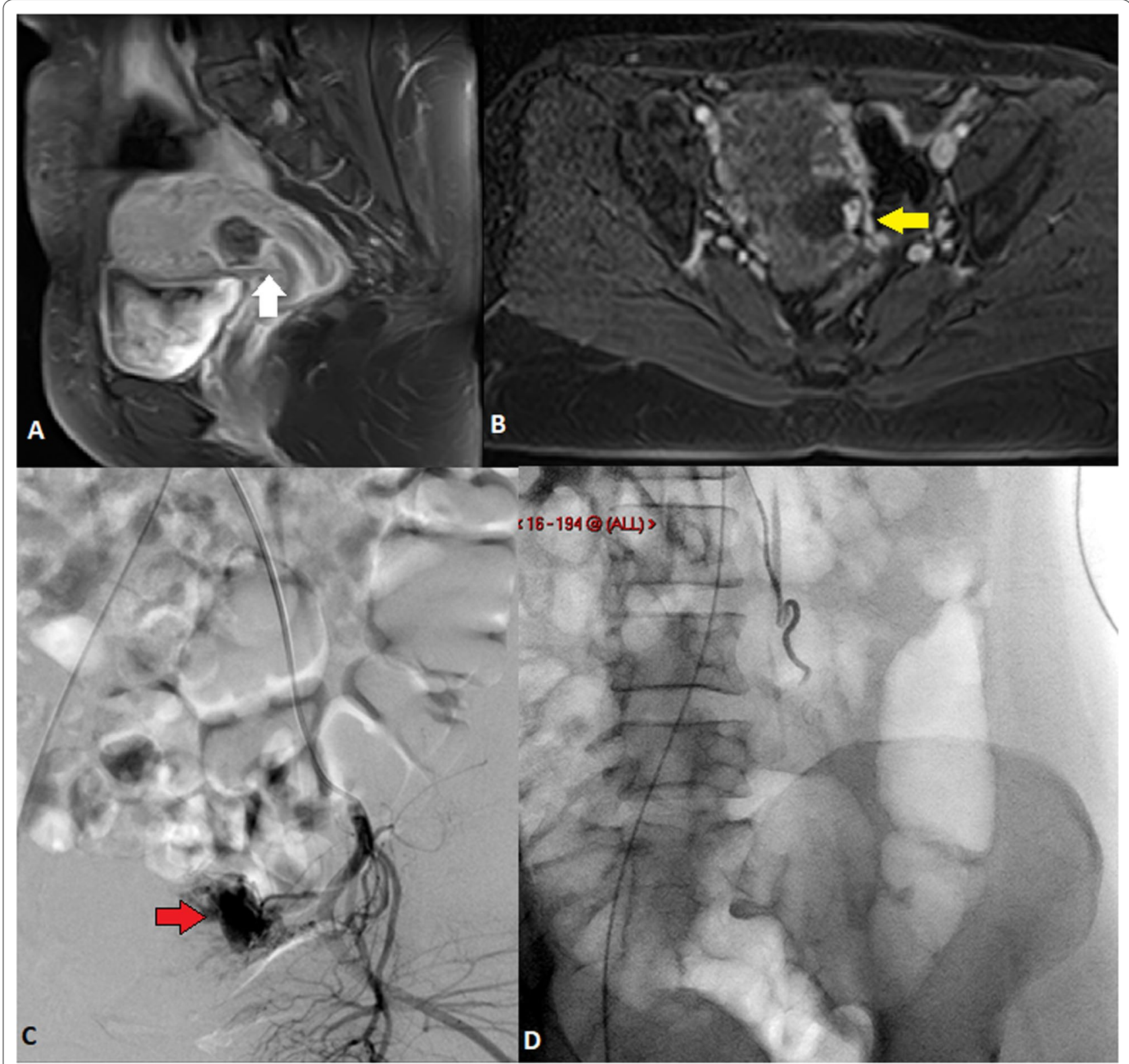

Fig. 6 Acquired uterine AVM and pseudoaneurysm in a 30-year-old. She had a history of previous two caesarian sections, therapeutic D\&C for GTC and presented with heavy vaginal bleeding for two months. A Dynamic post-contrast MRl, saggital image shows an abnormal signal area in the lower uterine body (white arrow) B same area in axial section shows a multiple abnormal flow voids over the uterine surface and a large vessel reaching up to the above described abnormal signal area (yellow arrow). C The pelvic arteriogram showed an AVM in the lower uterine body supplied by the left ovarian artery and bilateral uterine arteries. In addition, a $2 \mathrm{~cm}$ pseudoaneurysm of the left ovarian artery (red arrow) is also seen. D Successful PVA embolisation of vascular malformation and pseudoaneurysm in the uterus supplied by left ovarian and bilateral uterine arteries

\section{Abbreviations}

UAP: Uterine artery pseudoaneurysm; AVM: Arteriovenous malformations; AVF: Arteriovenous fistula; USG: Ultrasound; MRI: Magnetic resonance imaging; CT: Computed tomography; RPOCs: Retained products of conception; GTD: Gestational trophoblastic disease; PVA: Polyvinyl alcohol particles; Serum B hCG: Serum Beta human Chorionic Gonadotrophin hormone.

\section{Acknowledgements}

Not applicable.

\section{Authors' contributions}

LM conceived the study design, drafted the manuscript and complied the cases. AIR conceived the study idea and participated in its design and 
coordination. ZAK participated in drafting, critical revision of manuscript and case collection. SN helped in drafting the manuscript. All authors have read and approved the final version of the manuscript.

\section{Funding}

None.

\section{Availability of data and materials}

The data that support the findings of this study are available on request from the corresponding author.

\section{Declarations}

\section{Ethics approval and consent to participate}

This study was approved by Institutional Review Board and Ethics Committee (IRB \& EC) at Shifa International Hospital, Islamabad, Pakistan (reference No. IRB\#218-21).

\section{Consent for publication}

Not applicable.

\section{Competing interests}

The authors declare that they have no competing interests.

\section{Author details}

'Department of Diagnostic and Interventional Radiology, Shifa International Hospital, Islamabad, Pakistan. ${ }^{2}$ Department of Diagnostic and Interventional Radiology, Shifa International Hospital, Islamabad, Pakistan.

Received: 31 October 2021 Accepted: 17 December 2021

Published online: 04 January 2022

\section{References}

1. Sharma N, Ganesh D, Devi L, Srinivasan J, Ranga U (2013) Prompt diagnosis and treatment of uterine arcuate artery pseudoaneurysm: a case report and review of literature. JCDR 7(10):2303

2. Niola R, Cavaliere C, Marcello L, Maglione F, de Ritis R, Di Pietto F, Albano G, Nazzaro G, Sirimarco F, Mocerino C, Loreto M (2014) Role of interventional radiology in treating obstetric haemorrhages. Radiol Med (Torino) 119(8):607-615

3. Baba Y, Matsubara S, Kuwata T, Ohkuchi A, Usui R, Saruyama M, Nakata M, Suzuki M (2014) Uterine artery pseudoaneurysm: not a rare condition occurring after non-traumatic delivery or non-traumatic abortion. Arch Gynecol Obstet 290(3):435-440

4. Boi L, Savastano S, Beghetto M, Dall'Acqua J, Montenegro GM (2017) Embolisation of iatrogenic uterine pseudoaneurysm. Gynecol Minim Invasive Therapy 6(2):85-88

5. Zwimpfer TA, Monod C, Redling K, Willi H, Takes M, Fellmann-Fischer B, Manegold-Brauer G, Hösli I (2021) Uterine pseudoaneurysm on the basis of deep infiltrating endometriosis during pregnancy-a case report. BMC Pregnancy Childbirth 21(1):1-7

6. Subramaniam S, Nadarajan C, Aziz ME. Role of uterine artery embolisation in pseudoaneurysm of uterine artery: a rare cause of secondary postpartum hemorrhage. Cureus. 2018;10(2).

7. Zhu YP, Sun ZJ, Lang JH, Pan J (2018) Clinical characteristic and management of acquired uterine arteriovenous malformation. Chin Med J 131(20):2489

8. Khan S, Saud S, Khan I, Achakzai B. Acquired uterine arteriovenous malformation following dilatation and curettage treated with bilateral uterine artery embolisation: a case report. Cureus. 2019;11(3).

9. Polat P, Suma S, Kantarcý M, Alper F, Levent A (2002) Color Doppler US in the evaluation of uterine vascular abnormalities. Radiographics 22(1):47-53

10. Kwon JH, Kim GS (2002) Obstetric iatrogenic arterial injuries of the uterus: diagnosis with US and treatment with transcatheter arterial embolisation. Radiographics 22(1):35-46

11. Hoang VT, Van HA, Trinh CT, Pham NT, Huynh C, Ha TN, Huynh $\mathrm{PH}$, Nguyen HQ, Vo UG, Nguyen TT (2021) Uterine arteriovenous malformation: a pictorial review of diagnosis and management. J Endovasc Ther 18:15266028211025022

12. Gulati MS, Paul SB, Batra A, Sarma D, Dadhwal V, Nath J (2000) Uterine arteriovenous malformations: the role of intravenous 'dual-phase'CT angiography. Clin Imaging 24(1):10-14

13. Flors L, Leiva-Salinas C, Maged IM, Norton PT, Matsumoto AH, Angle JF, Hugo Bonatti MD, Park AW, Ahmad EA, Bozlar U, Housseini AM (2011) MR imaging of soft-tissue vascular malformations: diagnosis, classification, and therapy follow-up. Radiographics 31(5):1321-1340

14. Hashim H, Nawawi O (2013) Uterine arteriovenous malformation. MJMS 20(2):76

15. Vogelzang RL, Nemcek AA Jr, Skrtic Z, Gorrell J, Lurain JR (1991) Uterine arteriovenous malformations: primary treatment with therapeutic embolisation. J Vasc Interv Radiol 2(4):517-522

16. Ghai S, Rajan DK, Asch MR, Muradali D, Simons ME, TerBrugge KG (2003) Efficacy of embolisation in traumatic uterine vascular malformations. J Vasc Interv Radiol 14(11):1401-1408

17. Badawy SZ, Etman A, Singh M, Murphy K, Mayelli T, Philadelphia M (2001) Uterine artery embolisation: the role in obstetrics and gynaecology. Clin Imaging 25(4):288-295

\section{Publisher's Note}

Springer Nature remains neutral with regard to jurisdictional claims in published maps and institutional affiliations.

\section{Submit your manuscript to a SpringerOpen ${ }^{\circ}$ journal and benefit from:}

- Convenient online submission

- Rigorous peer review

- Open access: articles freely available online

- High visibility within the field

- Retaining the copyright to your article

Submit your next manuscript at springeropen.com 